\title{
Singular holomorphic functions for which all fibre-integrals are smooth
}

by D. BARLET (Vandœuvre-lès-Nancy) and H.-M. MAIRE (Genève)

Bogdan Ziemian in memoriam

\begin{abstract}
For a germ $(X, 0)$ of normal complex space of dimension $n+1$ with an isolated singularity at 0 and a germ $f:(X, 0) \rightarrow(\mathbb{C}, 0)$ of holomorphic function with $d f(x) \neq 0$ for $x \neq 0$, the fibre-integrals

$$
s \mapsto \int_{f=s} \varrho \omega^{\prime} \wedge \overline{\omega^{\prime \prime}}, \quad \varrho \in C_{\mathrm{C}}^{\infty}(X), \omega^{\prime}, \omega^{\prime \prime} \in \Omega_{X}^{n},
$$

are $C^{\infty}$ on $\mathbb{C}^{*}$ and have an asymptotic expansion at 0 . Even when $f$ is singular, it may happen that all these fibre-integrals are $C^{\infty}$. We study such maps and build a family of examples where also fibre-integrals for $\omega^{\prime}, \omega^{\prime \prime} \in \underline{\omega}_{X}$, the Grothendieck sheaf, are $C^{\infty}$.
\end{abstract}

0. Introduction. Let $(X, 0)$ be a germ of normal complex space of dimension $n+1$ with an isolated singularity at 0 and let $f:(X, 0) \rightarrow(\mathbb{C}, 0)$ be a germ of holomorphic function such that $d f(x) \neq 0$ for $x \neq 0$.

In a previous paper [B-M 99], we have explained how eigenvalues of the monodromy $M$ of $f$ acting on $H^{n}(F)$, where $F$ is the Milnor fibre of $f$, contribute to create poles of the meromorphic extension of the current $\lambda \mapsto$ $\Gamma(\lambda)^{-1} \int_{X}|f|^{2 \lambda} \square$. For eigenvalues different from 1, our results generalize those of the first author [B 84] for smooth $X$. But for the eigenvalue 1 of $M$, poles of the above current appear at negative integers if, and only if, 1 is also an eigenvalue of the monodromy of $f$ acting in the quotient $H^{n}(F) / J$, where $J$ is the image of the map $H^{n}(X \backslash\{0\}) \rightarrow H^{n}(F)$ induced by restriction. (See Example 3 for explicit computation of the image.) When this restriction is surjective (which implies $M=1$ and is therefore a very strong hypothesis), it follows that $\lambda \mapsto \int_{X}|f|^{2 \lambda} \square$ has only simple poles on the negative integers.

2000 Mathematics Subject Classification: 32C30, 32S30, 32S50, 58K15.

Key words and phrases: currents, fibre-integrals, Mellin transform, singularities. The authors acknowledge the support of the Swiss National Science Foundation. 
Using inverse Mellin transform, we deduce that, for all $\varphi \in C_{\mathrm{c}}^{\infty}(X)^{n, n}$, the fibre-integrals

$$
s \mapsto \int_{f=s} \varphi
$$

are of class $C^{\infty}$ (because

$$
\int_{X}|f|^{2 \lambda} \varphi \wedge \frac{d f}{f} \wedge \frac{d \bar{f}}{\bar{f}}=\int_{\mathbb{C}}|s|^{2 \lambda} \int_{f=s} \varphi \frac{d s}{s} \wedge \frac{d \bar{s}}{\bar{s}}
$$

by Fubini's theorem). In this situation, fibre-integrals of $C^{\infty}$ forms of type $(n, n)$ do not detect the singularity of the map $f: X \rightarrow \mathbb{C}$, that is, $X$ not smooth or $d f(0)=0$.

In a more general context, asymptotic expansions at 0 of functions (1) give rise to a finitely generated $\mathbb{C}[[s, \bar{s}]]$-module $\mathcal{M}$ (see Theorem 1 below). Because $X$ and $f$ have an isolated singularity at 0 , this module is generated by 1 and the asymptotic expansions of the following functions:

$$
s \mapsto \int_{f=s} \varrho \omega^{\prime} \wedge \overline{\omega^{\prime \prime}}, \quad \omega^{\prime}, \omega^{\prime \prime} \in \Omega_{X}^{n},
$$

where $\varrho \in C_{\mathrm{c}}^{\infty}(X)$ is equal to 1 near 0 . Indeed, for any integer $N>0$, there exist $\omega_{l}^{\prime}, \omega_{l}^{\prime \prime} \in \Omega_{X}^{n}$ and $L(N) \in \mathbb{N}$ such that

$$
\varphi-\sum_{l=1}^{L(N)} \varrho \omega_{l}^{\prime} \wedge \overline{\omega_{l}^{\prime \prime}}
$$

is flat of order $N$ at 0 ; because the coefficients of the non- $C^{\infty}$ terms of the asymptotic expansion are currents carried by 0 and because $\mathcal{M}$ is of finite type (cf. Theorem 1 below), the assertion follows.

When $f: X \rightarrow \mathbb{C}$ satisfies $J=H^{n}(F)$ the considerations above may be written briefly as

$$
\mathcal{M}=\mathbb{C}[[s, \bar{s}]] .
$$

In order to detect the singularity of the map $f: X \rightarrow \mathbb{C}$ with fibre-integrals, we then consider

$$
s \mapsto \int_{f=s} \varrho \omega^{\prime} \wedge \overline{\omega^{\prime \prime}}, \quad \omega^{\prime}, \omega^{\prime \prime} \in \underline{\omega}_{X}^{n},
$$

where $\underline{\omega}_{X}^{n}$ is the direct image sheaf on $X$ of holomorphic $n$-forms on the nonsingular part $X^{*}=X \backslash\{0\}$. The germs at 0 of these new fibre-integrals, to which we add the function 1 , have the structure of a $\mathbb{C}\{s, \bar{s}\}$-module; this module tensored by $\mathbb{C}[[s, \bar{s}]]$ gives a $\mathbb{C}[[s, \bar{s}]]$-module $\mathcal{N}$ containing $\mathcal{M}$. When $X$ is smooth, we have $\mathcal{N}=\mathcal{M}$ because, by Hartogs, $\underline{\omega}_{X}^{n}=\Omega_{X}^{n}$. In general we know that there exists an integer $\nu$ such that $|s|^{2 \nu} \mathcal{N} \subseteq \mathcal{M}$, because 
$f^{\nu} \underline{\omega}_{X}^{n} \subseteq \Omega_{X}^{n}$ (see Remark 1.2 of [B-M 99]). The trivial inclusion $\mathcal{M} \subseteq \mathcal{N}$ is strict in general as shown in Example 2.

Proposition 6 below shows that we may have $\mathcal{N}=\mathcal{M}=\mathbb{C}[[s, \bar{s}]]$ for a small but nonempty class of singular maps $f: X \rightarrow \mathbb{C}$. The invariant $\mathcal{N}$ is therefore not fine enough to detect the singularity of $f: X \rightarrow \mathbb{C}$. It is then natural to widen the class of fibre-integrals under consideration. To this end, if $\omega^{\prime}$ and $\omega^{\prime \prime}$ belong to $\underline{\omega}_{X}^{n+1}$ we look at

$$
s \mapsto\left(\int_{f=s} \varrho \frac{\omega^{\prime}}{d f} \wedge \frac{\overline{\omega^{\prime \prime}}}{d \bar{f}}\right) d s \wedge d \bar{s} .
$$

The $(1,1)$-form above is nothing but the direct image $f_{*}\left(\varrho \omega^{\prime} \wedge \overline{\omega^{\prime \prime}}\right)$. The asymptotic expansions of forms (4) generate a module $\mathcal{N}^{1,1}$ on $\mathbb{C}[[s, \bar{s}]]$. In case $X$ is smooth, this new module can be deduced from $\mathcal{N}=\mathcal{M}$ by means of the following relation:

$$
f_{*}\left(\varrho \underline{\omega}_{X}^{n+1} \wedge \overline{\Omega_{X}^{n}}\right) \subseteq d^{\prime} f_{*}\left(\varrho \Omega_{X}^{n} \wedge \overline{\Omega_{X}^{n}}\right) .
$$

Indeed, for $\omega^{\prime} \in \Omega_{X}^{n+1}$ and $\omega^{\prime \prime} \in \Omega_{X}^{n}$, we may write, using the holomorphic de Rham lemma,

$$
\omega^{\prime}=d^{\prime} \omega_{1} \quad \text { with } \omega_{1} \in \Omega_{X}^{n}
$$

Hence

$$
f_{*}\left(\varrho \omega^{\prime} \wedge \overline{\omega^{\prime \prime}}\right)=d^{\prime} f_{*}\left(\varrho \omega_{1} \wedge \overline{\omega^{\prime \prime}}\right)-f_{*}\left(d^{\prime} \varrho \wedge \omega_{1} \wedge \overline{\omega^{\prime \prime}}\right) .
$$

But because $\varrho$ is identically 1 near 0 , the direct image $f_{*}\left(d^{\prime} \varrho \wedge \omega_{1} \wedge \overline{\omega^{\prime \prime}}\right)$ belongs to $C_{\mathrm{c}}^{\infty}\left(\mathbb{C}^{*}\right)^{1,0} \subseteq d^{\prime} C^{\infty}(\mathbb{C})^{0,0}$ and hence $f_{*}\left(\varrho \omega_{1} \wedge \overline{\omega^{\prime \prime}}\right)$ belongs to $d^{\prime}\left(f_{*}\left(\varrho \Omega_{X}^{n} \wedge \bar{\Omega}_{X}^{n}\right)\right)$. Relation (5) implies that for $X$ smooth we have $\mathcal{N}^{1,1}=$ $d^{\prime} d^{\prime \prime} \mathcal{N}\left(=d^{\prime} d^{\prime \prime} \mathcal{M}\right)$. This equality does not hold in the example of $X=$ $\left\{x^{2}+y^{3}=z^{6}\right\}$ and $f=z$ (see (18) and Proposition 6) where $\mathcal{M}=\mathcal{N}=$ $\mathbb{C}[[s, \bar{s}]]$ and $\mathcal{N}^{1,1}$ contains $(d s \wedge d \bar{s}) / s \bar{s}$ ! When $X$ is singular, the holomorphic de Rham lemma is not valid and in fact relation (5) is no longer true.

It turns out that there exist very few maps for which $\mathcal{M}=\mathcal{N}=\mathbb{C}[[s, \bar{s}]]$ and $\mathcal{N}^{1,1}=\mathbb{C}[[s, \bar{s}]] d s \wedge d \bar{s}$. We describe the construction of a class of examples presenting that feature in Section 3 and conclude with very explicit singularities.

1. Asymptotic expansion of fibre-integrals. Let us start with a version of the asymptotic expansion theorem for fibre-integrals of forms of type (2), (3) or (4). We do not assume that $X$ and $f$ have an isolated singularity here.

TheOREM 1. Let $X$ be a reduced analytic space of pure dimension $n+1$ $\geq 2$ and let $f: X \rightarrow \mathbb{C}$ be a holomorphic function satisfying 
(i) $\operatorname{Sing}(X) \subseteq f^{-1}(0)$;

(ii) $d f(x)=0 \Rightarrow f(x)=0$ for $x \in X \backslash \operatorname{Sing}(X)$.

Then, for any $\varrho \in C_{\mathrm{c}}^{\infty}(X)$ and $\left(\omega^{\prime}, \omega^{\prime \prime}\right) \in \underline{\omega}_{X}^{n+p} \times \underline{\omega}_{X}^{n+q}$, where $p, q \in\{0,1\}$, the direct image $f_{*}\left(\varrho \omega^{\prime} \wedge \overline{\omega^{\prime \prime}}\right)$ is a $(p, q)$-current of class $C^{\infty}$ on $\mathbb{C}^{*}$, admitting, as $s \rightarrow 0$, an asymptotic expansion that belongs to

$$
\begin{array}{cl}
\bigoplus_{r \in R, k \in[0, n]} \mathbb{C}[[s, \bar{s}]]|s|^{2(r-\nu)} \log ^{k}|s|\left(\frac{d s}{s}\right)^{p} \wedge\left(\frac{d \bar{s}}{\bar{s}}\right)^{q} \quad \text { if } p+q>0, \\
\bigoplus_{r \in R, k \in[0, n]} \mathbb{C}[[s, \bar{s}]]|s|^{2(r-\nu)} \log ^{k}|s|+\mathbb{C}[[s, \bar{s}]]|s|^{-2 \nu} \quad \text { if } p+q=0,
\end{array}
$$

where $\nu$ is an integer, $R$ is a finite subset of $] 0,1] \cap \mathbb{Q}$ that only depends on $X, f$ and $\operatorname{supp} \varrho$. This asymptotic expansion may be differentiated termwise.

REMARK. In the second expression, terms of type $|s|^{-2 \nu} \log ^{k}|s|$ are not permitted if $k>0$.

Proof. By [B 78] the sheaf $\underline{\omega}_{X}^{n+p}$ is coherent and $\operatorname{supp} \underline{\omega}_{X}^{n+p} / \Omega_{X}^{n+p}$ is contained in $\operatorname{Sing}(X)$ for any $p$; the Nullstellensatz gives locally an integer $\nu$ such that $f^{\nu} \underline{\omega}_{X}^{n+p} \subseteq \Omega_{X}^{n+p} /$ torsion, using (i). There exist therefore two forms $\zeta^{\prime} \in \Omega_{X}^{n+p}$ and $\zeta^{\prime \prime} \in \Omega_{X}^{n+q}$ such that

$$
\omega^{\prime}=\zeta^{\prime} / f^{\nu} \text { and } \omega^{\prime \prime}=\zeta^{\prime \prime} / f^{\nu} .
$$

Because

$$
f_{*}\left(\varrho \omega^{\prime} \wedge \overline{\omega^{\prime \prime}}\right)(s)=f_{*}\left(\varrho \zeta^{\prime} / f^{\nu} \wedge \overline{\zeta^{\prime \prime}} / \bar{f}^{\nu}\right)(s)=\frac{1}{|s|^{2 \nu}} f_{*}\left(\varrho \zeta^{\prime} \wedge \overline{\zeta^{\prime \prime}}\right)(s),
$$

it is enough to prove that the asymptotic expansion of $f_{*}\left(\varrho \zeta^{\prime} \wedge \overline{\zeta^{\prime \prime}}\right)$ belongs to

$$
\begin{array}{ll}
\bigoplus_{r \in R, k \in[0, n]} \mathbb{C}[[s, \bar{s}]]|s|^{2 r} \log ^{k}|s|\left(\frac{d s}{s}\right)^{p} \wedge\left(\frac{d \bar{s}}{\bar{s}}\right)^{q} & \text { if } p+q>0, \\
\bigoplus_{r \in R, k \in[0, n]} \mathbb{C}[[s, \bar{s}]]|s|^{2 r} \log ^{k}|s|+\mathbb{C}[[s, \bar{s}]] & \text { if } p+q=0 .
\end{array}
$$

Let us desingularize $X$. We are reduced to proving the result when $X$ is nonsingular and $\omega^{\prime}, \omega^{\prime \prime}$ are holomorphic; therefore $\phi:=\varrho \omega^{\prime} \wedge \overline{\omega^{\prime \prime}}$ belongs to $C_{\mathrm{c}}^{\infty}(X)^{n+p, n+q}$. Using a partition of unity, we may even assume $X$ to be an open subset of $\mathbb{C}^{n+1}$.

Consider the case $p=q=1$. From the definition of direct images, when $\phi \in C_{\mathrm{c}}^{\infty}\left(X^{\prime}\right)^{n+1, n+1}$, where $X^{\prime}=X \backslash f^{-1}(0)$, we have

$$
\int_{X^{\prime}}|f|^{2 \lambda} \phi=\int_{\mathbb{C}^{*}}|s|^{2 \lambda} f_{*} \phi(s)
$$


Indeed, set $\psi(s)=|s|^{2 \lambda}$ in the relation $\left\langle f_{*} \phi, \psi\right\rangle=\left\langle\phi, f^{*} \psi\right\rangle$. It follows that for $\phi \in C_{\mathrm{c}}^{\infty}(X)^{n+1, n+1}$, the form $\left.f_{*} \phi\right|_{\mathbb{C}^{*}}$ is equal to $\mathfrak{M}^{-1}\left(\lambda \mapsto \int_{X}|f|^{2 \lambda} \phi\right)$ where $\mathfrak{M}$ is the complex Mellin transform defined by $\mathfrak{M} \alpha(\lambda)=\int_{\mathbb{C}}|s|^{2 \lambda} \alpha(s)$ for $\alpha \in C_{\mathrm{c}}^{\infty}\left(\mathbb{C}^{*}\right)^{1,1}$. It is well known that $\lambda \mapsto \int_{X}|f|^{2 \lambda} \phi$ admits a meromorphic extension to $\mathbb{C}$ with poles at strictly negative rationals contained in $-R-\mathbb{N}$ with some finite $R \subset] 0,1]$. Moreover $\lambda \mapsto \int_{X}|f|^{2 \lambda} \phi$ is rapidly decreasing on $\{\operatorname{Re} \lambda=$ const $\}$. Considering also the meromorphic extension of $\lambda \mapsto$ $\int_{X}|f|^{2 \lambda} \bar{f}^{m} \phi$ for $m \in \mathbb{Z}$ and taking the inverse Mellin transform we get the desired asymptotic expansion (see [B-M 89]).

In case $p=q=0$ instead of (6) we write

$$
\int_{X^{\prime}}|f|^{2 \lambda} \varphi \wedge \frac{d f}{f} \wedge \frac{d \bar{f}}{\bar{f}}=\int_{\mathbb{C}^{*}}|s|^{2 \lambda} f_{*} \varphi(s) \frac{d s}{s} \wedge \frac{d \bar{s}}{\bar{s}}, \quad \varphi \in C_{\mathrm{c}}^{\infty}(X)^{n, n} .
$$

When $f$ has only normal crossings we may write in an appropriate coordinate system

$$
f(z)=z_{0}^{\alpha_{0}} \ldots z_{n}^{\alpha_{n}}
$$

and so

$$
\begin{aligned}
\frac{d f}{f} \wedge \frac{d \bar{f}}{\bar{f}} & =\left(\alpha_{0} \frac{d z_{0}}{z_{0}}+\ldots+\alpha_{n} \frac{d z_{n}}{z_{n}}\right) \wedge\left(\alpha_{0} \frac{d \bar{z}_{0}}{\bar{z}_{0}}+\ldots+\alpha_{n} \frac{d \bar{z}_{n}}{\bar{z}_{n}}\right) \\
& =\sum \alpha_{j} \alpha_{k} \frac{d z}{z}{ }_{j} \wedge \frac{d \bar{z}_{k}}{\bar{z}_{k}} .
\end{aligned}
$$

When $j \neq k$, the form

$$
z \mapsto\left|z_{0}^{\alpha_{0}} \ldots z_{n}^{\alpha_{n}}\right|^{2 \lambda} \varphi(z) \wedge \frac{d z_{j}}{z_{j}} \wedge \frac{d \bar{z}_{k}}{\bar{z}_{k}}
$$

is integrable for $\operatorname{Re} \lambda \geq 0$; the pole at $\lambda=0$ of

$$
\lambda \mapsto \int|f|^{2 \lambda} \varphi \wedge \frac{d f}{f} \wedge \frac{d \bar{f}}{\bar{f}}
$$

is therefore created by terms of the type

$$
\int\left|z_{0}^{\alpha_{0}} \ldots z_{n}^{\alpha_{n}}\right|^{2 \lambda} \varphi(z) \wedge \frac{d z_{j}}{z_{j}} \wedge \frac{d \bar{z}_{j}}{\bar{z}_{j}}
$$

and it is simple. The result follows by taking the Mellin transform and (7).

The case $p=1, q=0$ is similar.

EXample 2. Computation of $\mathcal{M}$ and $\mathcal{N}$ for

$$
X=\left\{(x, y, z) \in \mathbb{C}^{3} \mid x y=z^{2}\right\} \quad \text { and } \quad f(x, y, z)=z .
$$

Using a Taylor expansion, we see that for $\varphi \in C_{\mathrm{c}}^{\infty}\left(\mathbb{C}^{3}\right)^{1,1}$,

$$
\int_{X, z=s} \varphi=\int_{x y=s^{2}} \varphi(x, y, s)=\sum_{p+q \leq N-1} s^{p} \bar{s}^{q} \int_{x y=s^{2}} \psi_{p q}+O\left(|s|^{N}\right)
$$


where $\psi_{p q} \in C_{\mathrm{c}}^{\infty}\left(\mathbb{C}^{2}\right)^{1,1}$. The asymptotic expansion of $\int_{x y=\sigma} \psi_{p q}$ belongs to the module $\mathbb{C}[[\sigma, \bar{\sigma}]] \oplus \mathbb{C}[[\sigma, \bar{\sigma}]]|\sigma|^{2} \log |\sigma|$ because the monodromy of the map $(x, y) \mapsto x y$ is the identity (see [B 85]). Therefore

$$
\mathcal{M}=\mathbb{C}[[s, \bar{s}]] \oplus \mathbb{C}[[s, \bar{s}]]|s|^{4} \log |s| .
$$

Take $\omega=(x d y-y d x) / z$. Then $\omega$ belongs to $\underline{\omega}_{X}^{1}$ because $x \omega$ and $y \omega$ are holomorphic. Standard computations give

$$
\int_{X, z=s} \varrho \omega \wedge \bar{\omega} \sim|s|^{2} \log |s|
$$

and so

$$
\mathcal{N}=\mathbb{C}[[s, \bar{s}]] \oplus \mathbb{C}[[s, \bar{s}]]|s|^{2} \log |s| .
$$

2. Occurrence of logarithmic terms. Let us recall the following consequence of Theorem 6.4 of [B-M 99] that guarantees the occurrence of a term $s^{m} \bar{s}^{m+j} \log |s|$ in the asymptotic expansion of fibre-integrals for $(n, n)$ forms. We assume that $(X, 0)$ is a germ of normal complex space of dimension $n+1$ with an isolated singularity at 0 and denote by $f:(X, 0) \rightarrow(\mathbb{C}, 0)$ a germ of holomorphic function such that $d f(x) \neq 0$ for $x \neq 0$.

Let $J$ be the image of the restriction map $H^{n}(X \backslash\{0\}) \rightarrow H^{n}(F)$, where $F$ is the Milnor fibre of $f$.

Theorem. Suppose $\omega$ is a holomorphic $n$-form on $X$ that satisfies

$$
d \omega=m \frac{d f}{f} \wedge \omega \quad \text { with some } m \in \mathbb{N} .
$$

Then the following two properties are equivalent:

(i) there exist $j \in \mathbb{Z}$ and $\omega^{\prime \prime} \in H^{0}\left(X, \Omega_{X}^{n}\right)$ such that the asymptotic expansion of the function $s \mapsto \int_{f=s} \varrho \omega \wedge \overline{\omega^{\prime \prime}}$ contains the term $s^{m} \bar{s}^{m+j} \log |s|$;

(ii) the class of $\omega / f^{m}$ in $H^{n}(F)^{M}$ does not belong to $J$.

REMARK. Using the decomposition of $\omega^{\prime \prime}$ in a Jordan basis of the GaussManin system of $f$, it is possible to choose $\omega^{\prime \prime}$ so as to have

$$
\int_{f=s} \varrho \omega \wedge \overline{\omega^{\prime \prime}} \equiv s^{m} \bar{s}^{m+j} \log |s|(\bmod \mathbb{C}[[s, \bar{s}]]),
$$

after increasing $j$ if necessary.

In the next example, we compute $J$ and $H^{n}(F)^{M}$.

EXAMPle 3. For the singularity $X=\left\{x^{2}+y^{3}+z^{6}=0\right\} \subset \mathbb{C}^{3}$ and $f: X \rightarrow \mathbb{C}$ given by $f(x, y, z)=x$ we have

$$
0 \varsubsetneqq J \varsubsetneqq H^{1}(F)^{M}=H^{1}(F)_{1} .
$$

Proof. Here, $n=1$ and the Milnor fibre of $f$ is $F=\left\{(1, y, z) \in \mathbb{C}^{3} \mid\right.$ $\left.y^{3}+z^{6}=-1\right\}$; it is therefore also the Milnor fibre of $g: \mathbb{C}^{2} \rightarrow \mathbb{C}$ given by 
$g(y, z)=y^{3}+z^{6}$. By Milnor, $\operatorname{dim} H^{1}(F)=10$. The corresponding monodromy $M_{g}$ is diagonal with eigenvalues $e^{2 i \pi 1 / 2}(2), e^{2 i \pi 2 / 3}, e^{2 i \pi 5 / 6}(2), e^{2 i \pi}$ $(2), e^{2 i \pi 7 / 6}(2), e^{2 i \pi 4 / 3}$ (the number in parentheses indicates multiplicity).

The commutative diagram

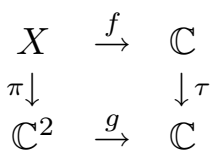

where $\pi(x, y, z)=(y, z)$ and $\tau(x)=x^{2}$ shows that $M_{f}=M_{g}^{2}$ and its eigenvalues are $e^{2 i \pi}(4), e^{2 i \pi 2 / 3}(3), e^{2 i \pi 4 / 3}(3)$. Here $H^{1}(F)^{M_{f}}=H^{1}(F)_{1}$ has dimension 4 and $\operatorname{dim} H^{1}\left(X^{*} \backslash X_{0}^{*}\right)=5$, where $X_{0}=\left\{x=0, y^{3}+z^{6}=0\right\}$. To check this last equality, remember that $H^{1}\left(X^{*} \backslash X_{0}^{*}\right) \cong H^{1}(F)_{1} \oplus \mathbb{C} \frac{d f}{f}$.

Let

$$
\omega_{1}=\frac{z d y-2 y d z}{x} \quad \text { and } \quad \omega_{2}=\frac{y z^{5} d y-2 y^{2} z^{4} d z}{x^{3}}=\frac{y z^{4}}{x^{2}} \omega_{1} .
$$

Then $\omega_{1}$ and $\omega_{2}$ give classes in $H^{1}\left(X \backslash X_{0}\right)$ which extend to $X^{*}$. The other three generators of $H^{1}\left(X \backslash X_{0}\right)$,

$$
\omega_{3}=\frac{y z}{x} \omega_{1}, \quad \omega_{4}=\frac{y^{3}}{x} \omega_{1} \quad \text { and } \quad \omega_{5}=\frac{d x}{x},
$$

do not "extend" to $X^{*}$.

REMARK. For the same singularity but with $f(x, y, z)=y$, it is easy to see that fibre-integrals of $C^{\infty}$ forms are not always $C^{\infty}$. As a consequence the wave front set of the integration current on $X \subset \mathbb{C}^{3}$ contains $\{0\} \times \mathbb{C}^{3}$ because it contains a cotangent vector $(0,0,0 ; 0,1,0)$ that does not belong to the closure of the conormal space to $X^{*}$.

3. A class of singularities with smooth fibre-integrals. In this section, we consider the following situation. Let $g \in \mathcal{O}_{\mathbb{C}^{n+1}}$ have an isolated singularity at $0, g(0)=0$. Denote by $M_{g}$ the monodromy of $g$ at 0 and suppose $M_{g}$ does not have the eigenvalue 1 , that is,

$$
M_{g}-1 \text { is invertible, }
$$

or, equivalently, the intersection form on $H^{n}(G)$, where $G$ is the Milnor fibre of $g$, is nondegenerate (see [A-G-Z-V], p. 410).

Assume also the existence of an integer $N>0$ such that

$$
M_{g}^{N}=1 \text {. }
$$

This last hypothesis implies that $M_{g}$ diagonalizes.

Let $\sigma(g)$ denotes the Arnold exponent of $g$ and $R(g) \subset] 0,1[$ its spectrum modulo 1. Hypothesis (9) yields

$$
N \cdot R(g) \subset \mathbb{N}^{*} .
$$


By classical results (cf. [B 85]), fibre-integrals with respect to $g$ have asymptotic expansions at 0 of the following type, for $\varrho \in C_{\mathrm{c}}^{\infty}\left(\mathbb{C}^{n+1}\right)$ equal to 1 in a neighbourhood of $0 \in \mathbb{C}^{n+1}$ :

$$
\begin{aligned}
& \eta^{\prime}, \eta^{\prime \prime} \in \Omega_{\mathbb{C}^{n+1}}^{n} \Rightarrow \int_{g=t} \varrho \eta^{\prime} \wedge \overline{\eta^{\prime \prime}} \in \sum_{r \in R(g)} \mathbb{C}[[t, \bar{t}]]|t|^{2 r}, \\
& \zeta^{\prime}, \zeta^{\prime \prime} \in \Omega_{\mathbb{C}^{n+1}}^{n+1} \Rightarrow \int_{g=t} \varrho \frac{\zeta^{\prime}}{d g} \wedge \frac{\overline{\zeta^{\prime \prime}}}{d \bar{g}} \in \sum_{r \in R(g)} \mathbb{C}[[t, \bar{t}]]|t|^{2 r-2} .
\end{aligned}
$$

There are no logarithmic terms because $1 \notin \operatorname{spec} M_{g}$ and all Jordan blocks of $M_{g}$ have size 1 .

Let us now define the analytic space $X$ and the holomorphic function $f$ we will study in this section:

$$
\begin{aligned}
& X=\left\{(x, s) \in \mathbb{C}^{n+2} \mid g(x)=s^{N}\right\}, \quad \text { where } g \text { satisfies (8) and (9), } \\
& f(x, s)=s .
\end{aligned}
$$

Observe that the hypersurface $X$ has an isolated singularity at 0 because $d f \wedge\left(d g-N s^{N-1} d s\right)=0$ implies $d s \wedge d g=0$.

The commutative diagram

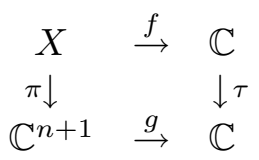

where $\pi(x, s)=x$ and $\tau(s)=s^{N}$, shows that the fibres of $f$ and $g$ are isomorphic because $f^{-1}(s)=g^{-1}\left(s^{N}\right) \times\{s\}$; it also explains why $M_{f}=$ $M_{g}^{N}=1$. On $X^{\prime}:=X \backslash\{s=0\}$, we have

$$
N \frac{d s}{s}=\frac{d g}{g} \text {. }
$$

As a consequence, for any $\eta \in \Omega_{\mathbb{C}^{n+1}}^{n}$ such that $d \eta=r \frac{d g}{g} \wedge \eta$, the following formula holds:

$$
d\left(\frac{\pi^{*} \eta}{s^{m}}\right)=(r N-m) \frac{d s}{s} \wedge \frac{\pi^{*} \eta}{s^{m}}, \quad m \in \mathbb{N} .
$$

For holomorphic forms on $X$ we use the obvious decomposition

$$
\begin{aligned}
\Omega_{X}^{n} & =\mathcal{O}_{X} \pi^{*} \Omega_{\mathbb{C}^{n+1}}^{n}+\mathcal{O}_{X} \pi^{*} \Omega_{\mathbb{C}^{n+1}}^{n-1} \wedge d s, \\
\Omega_{X}^{n+1} & =\mathcal{O}_{X} \pi^{*} \Omega_{\mathbb{C}^{n+1}}^{n+1}+\mathcal{O}_{X} \pi^{*} \Omega_{\mathbb{C}^{n+1}}^{n} \wedge d s .
\end{aligned}
$$

For the sheaves $\underline{\omega}_{X}^{n}$ and $\underline{\omega}_{X}^{n+1}$ we need a lemma.

LEMma 4. Under hypothesis $(\mathrm{H})$, we have

$$
\underline{\omega}_{X}^{n} \subseteq \frac{1}{s^{N-1}} \mathcal{O}_{X} \pi^{*} \Omega_{\mathbb{C}^{n+1}}^{n}, \quad \underline{\omega}_{X}^{n+1} \subseteq \frac{1}{s^{N-1}} \mathcal{O}_{X} \pi^{*} d x_{0} \wedge \ldots \wedge d x_{n} .
$$


Proof. Let $j=n$ or $n+1$. Any section of $\underline{\omega}_{X}^{j}$ near the origin belongs to $\Omega_{X}^{j}\left[s^{-1}\right]$ because there exists $\nu \in \mathbb{N}$ such that $s^{\nu} \underline{\omega}_{X}^{j}$ is contained in $\Omega_{X}^{j}$ modulo torsion. On the other hand, on $X^{\prime}$ we have

$$
d s=\frac{1}{N s^{N-1}} d g,
$$

by (12). So any section of $\underline{\omega}_{X}^{j}$ is a finite sum of elements of the type $\pi^{*} \alpha / s^{k}$ where $\alpha \in \Omega_{\mathbb{C}^{n+1}}^{j}$ and $k \in \mathbb{Z}$. Suppose $k \geq N$; because the sections of $\underline{\omega}_{X}^{j}$ have the trace property (see [B 78]), we get

$$
\operatorname{trace}_{\pi}\left(\frac{\pi^{*} \alpha}{s^{N}}\right)=N \frac{\alpha}{g} \in \Omega_{\mathbb{C}^{n+1}}^{j} .
$$

Hence $\alpha=g \beta$ with $\beta \in \Omega_{\mathbb{C}^{n+1}}^{j}$ and so $\pi^{*} \alpha / s^{k}=\pi^{*} \beta / s^{k-N}$. Iterating this process we are reduced to $k \leq N-1$, proving the inclusions.

REMARK. The second inclusion is in fact an equality.

Proposition 5. Assume $(\mathrm{H})$. Then for $(p, q) \in\{0,1\}$ we have

$$
\mathcal{M}^{p, q}=\mathbb{C}[[s, \bar{s}]] d s^{p} \wedge d \bar{s}^{q} .
$$

Proof. CASE 1: $p=q=0$. Thanks to (14) we only need to show that fibre-integrals for $\pi^{*} \eta^{\prime} \wedge \pi^{*} \eta^{\prime \prime}$ are $C^{\infty}$ for $\eta^{\prime}, \eta^{\prime \prime} \in \Omega_{\mathbb{C}^{n+1}}^{n}$. Indeed, the second term in (14) does not contribute and $\mathcal{O}_{X} \subseteq \mathbb{C}[[s]] \pi^{*} \mathcal{O}_{\mathbb{C}^{n+1}}$ explains how $\mathcal{O}_{X}$ coefficients are treated. But

$$
\int_{f^{-1}(s) \cap X} \varrho \pi^{*} \eta^{\prime} \wedge \pi^{*} \overline{\eta^{\prime \prime}}=\int_{g=s^{N}} \varrho \eta^{\prime} \wedge \overline{\eta^{\prime \prime}} \in \sum_{r \in R(g)} \mathbb{C}\left[\left[s^{N}, \bar{s}^{N}\right]\right]|s|^{2 r N} \subseteq \mathbb{C}[[s, \bar{s}]]
$$

by (11) and (10).

CASE 2: $p=q=1$. The term containing $d s$ in formula (14) produces a $C^{\infty}$ term after fibre-integration, from the first part of the proof. Now for $\zeta \in \Omega_{\mathbb{C}^{n+1}}^{n+1}$, we have

$$
\frac{\pi^{*} \zeta}{d s}=\frac{1}{s} \pi^{*}\left(\frac{N g \zeta}{d g}\right)=N s^{N-1} \pi^{*}\left(\frac{\zeta}{d g}\right)
$$

from (13) and hence

$$
\begin{aligned}
\int_{f^{-1}(s)} \varrho \frac{\pi^{*} \zeta^{\prime}}{d s} \wedge \frac{\pi^{*} \overline{\zeta^{\prime \prime}}}{d \bar{s}} & =N^{2}|s|^{2 N-2} \int_{g=s^{N}} \varrho \frac{\zeta^{\prime}}{d g} \wedge \frac{\overline{\zeta^{\prime \prime}}}{d \bar{g}} \\
& \in \sum_{r \in R(g)} \mathbb{C}\left[\left[s^{N}, \bar{s}^{N}\right]\right]|s|^{2 r N-2} ;
\end{aligned}
$$

this fibre-integral is $C^{\infty}$ because $N \sigma(g)-1 \geq 0$ from $N \sigma(g) \in \mathbb{N}^{*}$.

Other cases are left to the reader. 
REMARKS. 1) The cutoff function $\varrho$ need not be compactly supported in $s$, that is why it only depends on $x$ in the above calculations. In fact the $f$-proper forms and the compactly supported ones give the same asymptotic expansions modulo $\mathbb{C}[[s, \bar{s}]]$.

2) Proposition 5 and Corollary 6.5 of [B-M 99] show that $\operatorname{dim} H^{n}\left(X^{*}\right)=$ $\operatorname{dim} H^{n}(F)$. In our situation $(\mathrm{H})$, this dimension is easily computable because $F$ is isomorphic to the Milnor fibre of $g$.

Proposition 6. Under hypothesis $(\mathrm{H})$, the following implications hold:

(a) $N \sigma(g) \geq N-1 \Rightarrow \mathcal{N}_{f}=\mathbb{C}[[s, \bar{s}]$;

(b) $\sigma(g)>1 \Leftrightarrow \mathcal{N}_{f}^{1,1}=\mathbb{C}[[s, \bar{s}]] d s \wedge d \bar{s}$.

The converse of (a) is true for quasi-homogeneous $g$.

Remark. Because $\sigma(g)$ is not an integer, $\sigma(g)>1$ is equivalent to $\sigma(g)$ $\geq 1$. On the other hand, because $N \sigma(g)$ is an integer, $\sigma(g) \geq 1$ is equivalent to $\sigma(g)>(N-1) / N$.

Proof of Proposition 6. (a) $(\Rightarrow)$ Let $\eta^{\prime}, \eta^{\prime \prime} \in \underline{\omega}_{X}^{n}$. By Lemma 4, there exist $\eta_{j}^{\prime}, \eta_{k}^{\prime \prime} \in \Omega_{\mathbb{C}^{n+1}}^{n}$ such that

$$
\eta^{\prime}=\frac{1}{s^{N-1}} \sum_{j=0}^{\infty} s^{j} \pi^{*} \eta_{j}^{\prime}, \quad \eta^{\prime \prime}=\frac{1}{s^{N-1}} \sum_{k=0}^{\infty} s^{k} \pi^{*} \eta_{k}^{\prime \prime} .
$$

Therefore

$$
\begin{aligned}
\int_{f^{-1}(s) \cap X} \varrho \pi^{*} \eta^{\prime} \wedge \pi^{*} \overline{\eta^{\prime \prime}} & =\sum_{j, k \geq 0} \int_{g=s^{N}} \varrho s^{j-N+1} \bar{s}^{k-N+1} \eta_{j}^{\prime} \wedge \overline{\eta_{k}^{\prime \prime}} \\
& \in \mathbb{C}[[s, \bar{s}]]|s|^{2 N \sigma(g)-2 N+2} \subseteq \mathbb{C}[[s, \bar{s}]] .
\end{aligned}
$$

$(\mathrm{a})(\Leftarrow)$ When $g$ is quasi-homogeneous, from $[\mathrm{L}]$ we get the existence of $\omega \in \Omega_{\mathbb{C}^{n+1}}^{n}$ such that

$$
\int_{g=t} \varrho \omega \wedge \bar{\omega}=|t|^{2 \sigma(g)}+o\left(|t|^{2 \sigma(g)}\right) .
$$

It is possible to choose $\omega$ such that

$$
d \omega=\sigma(g) \frac{d g}{g} \wedge \omega
$$

Consider $\eta=\left(1 / s^{N-1}\right) \pi^{*} \omega$; we check $\eta$ belongs to $\underline{\omega}_{X}^{n}$. By [B 78], it is enough to see that for all $j \in[0, N-1]$,

$$
\operatorname{trace}_{\pi}\left(\frac{s^{j}}{s^{N-1}} \pi^{*} \omega\right) \in \Omega_{\mathbb{C}^{n+1}}^{n} \quad \text { and } \quad \operatorname{trace}_{\pi}\left(\frac{s^{j}}{s^{N-1}} d s \wedge \pi^{*} \omega\right) \in \Omega_{\mathbb{C}^{n+1}}^{n+1} .
$$

The first trace vanishes for $j<N-1$, and it is equal to $N \omega$ when $j=N-1$. The second trace is nonzero only for $j=N-2$ and then it is equal to $\frac{d g}{g} \wedge \omega$. 
Relation (16) implies $\eta \in \underline{\omega}_{X}^{n}$.

Integrating along fibres, we get, from (15),

$$
\int_{f^{-1}(s) \cap X} \varrho \eta \wedge \bar{\eta}=|s|^{2 N \sigma(g)-2 N+2}(1+o(1)) .
$$

In order that this integral be $C^{\infty}$, we must have $N \sigma(g) \geq N-1$.

$(\mathrm{b})(\Rightarrow)$ For $\zeta \in \Omega_{\mathbb{C}^{n+1}}^{n+1}$, we have by (12),

$$
\frac{1}{s^{N-1}} \frac{\pi^{*} \zeta}{d s}=\frac{1}{s^{N}} \pi^{*}\left(\frac{N g \zeta}{d g}\right)=N \pi^{*}\left(\frac{\zeta}{d g}\right) .
$$

Taking fibre-integrals gives

$$
\int_{f^{-1}(s)} \varrho \frac{\pi^{*} \zeta^{\prime}}{d s} \wedge \frac{\pi^{*} \overline{\zeta^{\prime \prime}}}{d \bar{s}}=N^{2} \int_{g=s^{N}} \varrho \frac{\zeta^{\prime}}{d g} \wedge \frac{\overline{\zeta^{\prime \prime}}}{d \bar{g}} \in \sum_{r \in R(g)} \mathbb{C}\left[\left[s^{N}, \bar{s}^{N}\right]\right]|s|^{2 N(r-1)} ;
$$

this fibre-integral is $C^{\infty}$. It remains to use $\mathbb{C}[[s, \bar{s}]]$-linearity and Lemma 4 .

$(\mathrm{b})(\Leftarrow)$ Following $[\mathrm{L}]$, take a holomorphic $(n+1)$-form $\Omega$ on $\mathbb{C}^{n+1}$ such that

$$
\int_{g=t} \varrho \frac{\Omega}{d g} \wedge \frac{\bar{\Omega}}{d \bar{g}}=|t|^{2 \sigma(g)-2}+o\left(|t|^{2 \sigma(g)-2}\right) .
$$

With $\zeta:=\frac{1}{N s^{N-1}} \pi^{*} \Omega \in \underline{\omega}_{X}^{n+1}$ we have

$$
\int_{f^{-1}(s) \cap X} \varrho \frac{\zeta}{d s} \wedge \frac{\bar{\zeta}}{d \bar{s}}=|s|^{2 N(\sigma(g)-1)}(1+o(1)) .
$$

If this integral is $C^{\infty}$ then $\sigma(g) \geq 1$.

4. Explicit examples. We present here explicit examples of singularities $X$ and functions $f$ for which all fibre-integrals are $C^{\infty}$; integration of forms in $\underline{\omega}_{X}^{n+1}$ is allowed.

To fulfill conditions (8) and (9), we look for Fermat's singularities

$$
g(x)=x_{0}^{p_{0}}+\ldots+x_{n}^{p_{n}}
$$

where $p_{0}, \ldots, p_{n}$ are integers $\geq 2$ that satisfy

$$
\frac{a_{0}}{p_{0}}+\ldots+\frac{a_{n}}{p_{n}} \notin \mathbb{N}
$$

for all $a_{j} \in \mathbb{N}$ with $0<a_{j}<p_{j}$. We take $N=\operatorname{lcm}\left(p_{0}, \ldots, p_{n}\right)$ in (9). A sufficient condition for (17) is

$$
\exists j \in[0, n] \text { such that }\left(p_{j}, p_{k}\right)=1, \forall k \neq j .
$$

For $n$ even, the following condition is also sufficient:

$$
\forall j, \forall k: \quad\left(p_{j}, p_{k}\right)=2 \text { if } j \neq k .
$$


CASE $n=1$. Condition (17) is equivalent to $\left(p_{0}, p_{1}\right)=1$ and so $N=p_{0} p_{1}$. The smallest values of $p_{0} \leq p_{1}$ are 2,3 , so that

$$
\frac{1}{p_{0}} \leq \frac{1}{2}, \quad \frac{1}{p_{1}} \leq \frac{1}{3}, \quad \frac{1}{N} \leq \frac{1}{6} .
$$

Hence

$$
\frac{1}{p_{0}}+\frac{1}{p_{1}}+\frac{1}{N} \leq 1 \quad \text { or } \quad \frac{1}{p_{0}}+\frac{1}{p_{1}} \leq \frac{N-1}{N}
$$

and the inequalities are strict if $p_{0}>2$ or $p_{1}>3$. Hence the only $X$ for which Proposition 6 applies is

$$
X=\left\{x_{0}^{2}+x_{1}^{3}=s^{6}\right\} .
$$

CASE $n=2$. We take $p_{0} \leq p_{1} \leq p_{2}$ and notice that

$$
\frac{1}{p_{0}}+\frac{1}{p_{1}}+\frac{1}{p_{2}} \geq \frac{N-1}{N}
$$

with $N=\operatorname{lcm}\left(p_{0}, p_{1}, p_{2}\right)$ may be satisfied, because $N \geq p_{2}$, only if

$$
p_{2} \leq \frac{2 p_{0} p_{1}}{p_{0} p_{1}-p_{0}-p_{1}} .
$$

This remark enables us to easily eliminate many values of $p_{0}, p_{1}, p_{2}$ satisfying (17).

\begin{tabular}{ccccclc}
\hline$p_{0}$ & $p_{1}$ & $p_{2}$ & $N$ & $\sigma(g)$ & & $\frac{N-1}{N}$ \\
\hline 2 & 2 & $2 k$ & $2 k$ & $\frac{2 k+1}{2 k}$ & $>$ & $\frac{2 k-1}{2 k}$ \\
2 & 2 & $2 k+1$ & $2 k+1$ & $\frac{2 k+2}{2 k+1}$ & $>$ & $\frac{2 k}{2 k+1}$ \\
2 & 3 & 3 & 6 & $\frac{5}{6}=$ & $\frac{5}{6}$ \\
2 & 3 & 4 & 12 & $\frac{13}{12}$ & $>$ & $\frac{11}{12}$ \\
2 & 3 & 5 & 30 & $\frac{31}{30}$ & $>$ & $\frac{29}{30}$ \\
2 & 3 & 7 & 42 & $\frac{41}{42}=\frac{41}{42}$ \\
2 & 3 & 8 & 24 & $\frac{23}{24}$ & $=\frac{23}{24}$ \\
2 & 3 & 9 & 18 & $\frac{17}{18}$ & $=\frac{17}{18}$ \\
2 & 4 & 5 & 20 & $\frac{19}{20}$ & $=\frac{19}{20}$ \\
2 & 5 & 5 & 10 & $\frac{9}{10}$ & $=\frac{9}{10}$ \\
3 & 3 & 4 & 12 & $\frac{11}{12}$ & $=\frac{11}{12}$ \\
\hline
\end{tabular}

In the table above, we give all triples $p_{0}, p_{1}, p_{2}$ for which Proposition 6 applies, that is, (17) and $\sigma(g) \geq(N-1) / N$ hold. So there are only few examples where $\sigma(g)>1$, i.e., examples for which all fibre-integrals are smooth. 
REMARK. Using the Thom-Sebastiani result, it is easy to see that if $g$ and $N$ satisfy conditions (8) and (9), then the function $G$ defined by $G\left(x, y, z_{0}, \ldots, z_{n}\right)=x^{2}+y^{2}+g\left(z_{0}, \ldots, z_{n}\right)$ gives also an example with the same $N$. So the first two series of examples in the table come from trivial examples in dimension $1(n=0)$.

Application. The wave front set of the integration current on the quadratic cone $X=\left\{x_{0}^{2}+\ldots+x_{n}^{2}=s^{2}\right\}$, for $n$ even, is equal to the closure of the conormal space to $X^{*}$. For $n$ odd this wave front set contains $\{0\} \times \mathbb{C}^{n+2}$. This follows, for $n$ even, from the fact that fibre-integrals with respect to $\xi_{0} x_{0}+\ldots+\xi_{n} x_{n}+\eta s$ are $C^{\infty}$ if $\xi_{0}^{2}+\ldots+\xi_{n}^{2} \neq \eta^{2}$ because a linear change of coordinates leaving the cone fixed reduces to our situation. Same argument for $n$ odd.

\section{References}

[A-G-Z-V] V. I. Arnold, S. M. Gusein-Zade and A. N. Varchenko, Singularities of Differentiable Maps II, Birkhäuser, 1988.

[B 78] D. Barlet, Le faisceau $\omega_{X}$ sur un espace analytique $X$ de dimension pure, in: Lecture Notes in Math. 670, Springer, 1978, 187-204.

[B 84] -, Contribution effective de la monodromie aux développements asymptotiques, Ann. Sci. Ecole Norm. Sup. 17 (1984), 293-315.

[B 85] - Forme hermitienne canonique sur la cohomologie de la fibre de Milnor d'une hypersurface à singularité isolée, Invent. Math. 81 (1985), 115-153.

[B-M 89] D. Barlet and H.-M. Maire, Asymptotic expansion of complex integrals via Mellin transform, J. Funct. Anal. 83 (1989), 233-257.

[B-M 99] - - - Poles of the current $|f|^{2 \lambda}$ over an isolated singularity, Internat. J. Math. (2000) (to appear).

[L] F. Loeser, Quelques conséquences locales de la théorie de Hodge, Ann. Inst. Fourier (Grenoble) 35 (1985), no. 1, 75-92.

Université H. Poincaré (Nancy I) et Institut Universitaire de France Institut E. Cartan UHP/CNRS/INRIA, UMR 7502 Boîte postale 239

F-54506 Vandœuvre-lès-Nancy, France E-mail: barlet@iecn.u-nancy.fr
Section de Mathématiques Université de Genève Case postale 240 CH-1211 Genève 24, Switzerland E-mail: henri.maire@math.unige.ch 